\title{
Renal cell carcinoma lung metastases treated by radiofrequency ablation integrated with systemic treatments: over 10 years of experience
}

Alexis Gonnet ${ }^{1,2}$, Laura Salabert ${ }^{3,4}$, Guilhem Roubaud ${ }^{4}$, Vittorio Catena', Véronique Brouste ${ }^{5}$, Xavier Buy ${ }^{1}$, Marine Gross Goupil ${ }^{3}$, Alain Ravaud ${ }^{3}$ and Jean Palussière ${ }^{1 *}$

\begin{abstract}
Background: To determine safety and efficacy of radiofrequency ablation (RFA) for local treatment of lung metastases of renal cell carcinoma (RCC), sequenced or combined with systemic treatments.

Methods: Retrospectively, we studied 53 patients treated by RFA for a maximum of six lung metastases of RCC. The endpoints were local efficacy, overall (OS), disease-free (DFS), pulmonary progression-free (PPFS) and systemic treatment-free (STFS) survivals, complications graded by the CTCAE classification and factors associated with survivals. Potential factors analysed were: clinical and pathological data, tumoral staging of TNM classification, primary tumor histology, Fuhrman's grade, age, number and size of lung metastases and extra-pulmonary metastases pre-RFA.

Results: One hundred metastases were treated by RFA. Median follow-up time was 61 months (interquartile range 90-34). Five-year OS was 62\% (95\% confidence interval (CI): 44-75). Median DFS was 9.9 months (95\% Cl: 6-16). PPFS at 1 and 3 years was 58.9\% (95\%Cl: 44.1-70.9) and 35.2\% (95\%Cl: 21.6-49.1), respectively. We observed 3\% major complications (grade 3 and 4 of CTCAE classification). Local efficacy was 91\%. Median STFS was 28.3 months. Thirteen patients (25\%) with lung recurrence could be treated by another RFA. T3/T4 tumors had significantly worse OS, PPFS and STFS. Having two or more lung metastases increased the risk of pulmonary progression more than threefold.

Conclusion: Integrated to systemic treatment strategy, RFA is safe and effective for the treatment strategy of lung metastasis from RCC with good OS and long systemic treatment-free survival. RFA offers the possibility of repeat procedures, with low morbidity.
\end{abstract}

Keywords: Renal cell carcinoma, Metastases, Lung metastases, Radiofrequency ablation, Local treatment, Oligometastasis

\footnotetext{
* Correspondence: j.palussiere@bordeaux.unicancer.fr

${ }^{1}$ Department of Radiology, Institut Bergonié, 229 cours de I'Argonne, 33000

Bordeaux, France

Full list of author information is available at the end of the article
}

(c) The Author(s). 2019 Open Access This article is distributed under the terms of the Creative Commons Attribution 4.0 International License (http://creativecommons.org/licenses/by/4.0/), which permits unrestricted use, distribution, and reproduction in any medium, provided you give appropriate credit to the original author(s) and the source, provide a link to the Creative Commons license, and indicate if changes were made. The Creative Commons Public Domain Dedication waiver (http://creativecommons.org/publicdomain/zero/1.0/) applies to the data made available in this article, unless otherwise stated. 


\section{Background}

Renal cell carcinoma (RCC) is the seventh most common solid cancer, with an incidence of 14.5 per 100,000 people $[1,2]$. The most frequent site of metastasis and recurrence is the lung, with more than $50-60 \%$ of patients developing lung metastases [3]. Recently, both targeted therapy and immunotherapy have broadened treatment options and improved prognosis [4-6], but systemic treatments remain expensive and targeted therapies are known to affect quality of life by their related toxicities.

Percutaneous image-guided thermal ablation is increasingly used to treat metastatic disease. Thermal ablation like radiofrequency (RFA) is effective with few complications and repeatable without distorting lung function [7]. RFA for the treatment of RCC lung metastases and repeat RFA have rarely been reported [8,9]. As a local treatment, lung metastasectomy for RCC remains the reference curative treatment while stereotactic body radiotherapy (SBRT) is another option and has shown better local control than conventional fractionated radiotherapy [10]. RCC is considered less radioresponsive than other histologies.

The aim of this study was to evaluate results of RFA of lung metastases from kidney cancer in terms of local ablation efficacy, overall survival (OS), disease-free survival (DFS) and systemic treatment-free survival (STFS), and to define the factors associated with better outcomes.

\section{Methods}

The aim of this study was to investigate local efficacy (absence of local recurrence), survivals and occurrence of complications after local treatment by RFA for RCC lung metastases treated over time at our institution, as well as the time without systemic treatment in order to report a potential effect of RFA on systemic treatment sparing. We also analyse factors associated with worse overall survival (OS), disease-free survival (DFS) and pulmonary progression-free (PPFS) survival.

This retrospective study included 53 patients (100 metastasis) treated in 65 sessions over 11 years at our large tertiary care centre. Patients with metastatic RCC (mRCC) were treated consecutively by RFA and registered in a database. Death notification or last patient follow-up news was obtained for all patients. This study was approved by the Institutional Review Board (Clinical Research Committee Institut Bergonié).

The decision to perform RFA was made after multidisciplinary discussion between radiologists, medical oncologists, radiation oncologists and surgeons. The radiologist informed the patient of the risks, possible complications, and benefits of the procedure. Limitations of and contraindications to RFA were represented mainly by the location of the tumor $(<1 \mathrm{~cm}$ from the hilum) and by the size of the tumor $(>40 \mathrm{~mm}$ ). Pulmonary lesions with characteristics of new or enlarging metastases in patients with known RCC were considered as sufficient for diagnosis of lung metastases without recourse to biopsy. Biopsies were performed in uncertain cases.

Patients either had no extra-pulmonary metastases, or if extra-pulmonary metastases were present, they were controlled by previous surgery or radiotherapy.

\section{Radiofrequency ablation technique and follow-up}

RFA was performed under helical CT guidance and under general anaesthesia. RFA used multiline expendable electrodes (LeVeen; Boston Scientific), with 3-, 3.5- or 4-cm array diameter when fully expanded.

Patients were followed up with whole-body CT imaging with intravenous contrast administration at 3, 6, 9 and 12 months and then every 6 months. Stability or any decrease in size was considered as complete treatment. An incomplete treatment was defined as regrowth, enlargement, or appearance of any irregular, nodular, or eccentric focus at the margin of the ablation zone by imaging. When a local failure was depicted, the patient file was reviewed by the multidisciplinary tumor group to determine the best option.

\section{Complications}

All treatment-related complications were categorized in accordance with the standardized Common Terminology Criteria for Adverse Events (CTCAE), version 4.0, of the National Cancer Institute.

\section{Statistical methods}

Median follow-up time was calculated by the inverse Kaplan-Meier technique and survivals by the KaplanMeier technique estimate rate were reported with their 95\%CI. OS was calculated as the time between RFA and the date of death or last news.

DFS was calculated as the time between RFA and recurrence. Recurrences in the ablation zone, pulmonary out of the ablation zone and extra-pulmonary were considered as events. Patients alive or dead without events were censored. In PPFS any new lung tumor either distant or a recurrence on RFA treated lesions was considered. PPFS was calculated as the time between RFA and any pulmonary recurrence, patients alive or dead without pulmonary events were censored. Local recurrencefree survival (LRFS) was defined as the time between RFA and any recurrence on treated lesions, patients without any evolution of treated lesions were censored. The local primary success rate (local efficacy) was defined as the percentage of tumors that were successfully eradicated following the initial procedure or a defined course of treatment. 
Free survival without systemic treatment (STFS) was defined as the time interval between RFA and the resuming ST or death without resuming the ST. Patients alive without the resumption of systemic treatment were censored at the date of last news.

We analysed: sex, age at RFA ( $\leq />60$ years), time interval between primary tumor and metastasis (concomitant or delayed), extra-thoracic metastases (yes/no), TNM classification $(\leq />2)$, Fuhrman grade $(\leq />2)$, size of the largest pulmonary lesion $(\leq />20 \mathrm{~mm})$, and number of locations $(1,2, \geq 3)$ as factors potentially associated with OS, DFS, PPFS and STFS with probability of Kaplan-Meier and log-rank test. All factors tested in univariate analysis were included in a multivariable backwards stepwise Cox manually regression model after verification of the proportionality hypothesis with the Shoendfeld test. SAS software, version 9.4, was used.

\section{Results}

\section{Population characteristics}

Thirty-four men and 19 women were included (Table 1), all of them treated with prior nephrectomy. One hundred metastases were treated by RFA. Median follow-up time was 61 months (interquartile range 90-34). Median number of metastases treated per patient was two (interquartile range 1-3). Median metastasis size was $12 \mathrm{~mm}$ (interquartile range $8-19 \mathrm{~mm}$ ). Median time between primary tumor and first metastasis was 22 months (interquartile range $7-53$ months).

\section{Recurrence and tumor progression}

Overall, 13 (25\%) patients with lung progression received a secondary RFA.

Nine local recurrences for nine patients occurred. The local efficacy percentage was $91 \%$ by lesion and $83 \%$ by patient. Seven of the nine local recurrences received a successful repeat ablation and achieved secondary local control (98\%). The remaining two were not able to receive secondary ablation due to extrapulmonary progressive disease (mediastinal nodal involvement).

One and 3- year LRFS were 93.8\% (95\%CI 82.0-97.9) and $82.6 \%$ (95\%CI 66.0-91.6) respectively.

\section{Survivals (Fig. 1)}

Median follow-up time was 60.8 months (95\% confidence interval [CI] 47.7-82.7). Median OS was not reached. OS at 1,3 and 5 years was $94.0 \%$ (95\%CI [82.6-98.0]), $74.5 \%$ (95\%CI [58.5-85.1]) and 61.8\% (95\%CI [44.0-75.3]), respectively. Median DFS was 9.9 months (95\%CI [6-16.4]) with DFS at 1 and 3 years of $40.3 \%$ (95\%CI [27.0-53.3]) and $18.0 \%$ (95\%CI [8.6-30.1]) respectively. Median PPFS was 21.7 months (95\%CI [10.1-34.4]) with PPFS at 1 and 3 years of $58.9 \%$ (95\%CI [44.1-70.93]) and 35.2\% (95\%CI [21.6-49.1]) respectively. Forty-two patients presented with progression in a median time interval of 8.5 months [interquartile range 3.9-20.8]. Of them, 24 were subsequently ST-free in a median time interval of 14.1 months [Interquartile range 6.1-24.8]. Median STFS was 28.3 months (95\% CI [17.8-83.1]).

\section{Complications (Table 2)}

Immediate complications occurred in 49 (75.4\%) of the 65 procedures, some were only image findings without clinical complaints. Pneumothorax was the most frequent and expected complication, and 26 of 39 pneumothoraces were drained. The chest tube when necessary was removed after 1 or 2 days in the absence of recurrent pneumothorax excepted in one patient in whom a prolonged chest tube drainage was needed (8 days hospitalization). In 13 of the procedures, the pneumothorax was minor and no treatment was required.

Only 3 complications were Grade 3-4 (pneumothorax requiring prolonged hospitalization; pain limiting daily activities; and a diaphragmatic hernia) (previously described) [11]. No procedural-related deaths were reported. The median duration of hospitalization was 3 days (range 1-8).

\section{Prognostic factors}

At univariate analysis, tumoral staging of TNM classification of the primary RCC $(P=0.031)$ and largest pulmonary metastasis size $>20 \mathrm{~mm} \quad(P=0.019)$ were significant factors for OS. All factors tested in univariate analysis were included in a multivariable model and only T3/T4 TNM classification was identified as significantly associated with poorer OS (HR 3.55, 95\%CI 1.05-12.1, $P=0.042$ ) (Table 3).

No associations were identified between DFS and potential factors in univariate analyses, neither in a multivariable analysis.

At univariate analysis, tumoral staging of TNM classification of the primary RCC was a significant factor for PPFS $(P=0.024)$, as was number of locations $(P=0.007)$. All factors tested in univariate analysis were included in a multivariable model and both a T3/4 classification $(\mathrm{HR}=$ 3.5, 95\%CI [1.3-9.3], $P=0.048)$, multiple lung metastases (2 metastases, HR $=3.1,95 \% \mathrm{CI}[1.1-8.7], P=0.032$; 3 metastases, $\mathrm{HR}=3.4,95 \% \mathrm{CI}[1.3-9.0], P=0.013)$ and sex (women $\mathrm{HR}=2.7,95 \% \mathrm{CI}[1.04-7.2], P=0.042$ ) were associated with significantly worse PPFS.

At univariate analysis, tumoral staging (T) of TNM classification of the primary RCC was the only significant factor for STFS $(P=0.0469)$. All factors tested in univariate analysis were included in a multivariable model and only T3/T4 TNM classification was identified as significantly associated with poorer STFS (HR 2.33, 95\%CI 0.9-5.5, $P=0.053$ ). 
Table 1 Population $(n=53)$ and Tumor $(n=100)$ characteristics

\begin{tabular}{ll}
\hline Characteristic & $\mathrm{N}(\%)$ \\
\hline Patient Sex & \\
Male & $34(64.2)$ \\
Female & $19(35.9)$ \\
Patient age at diagnostic (years) & \\
Median (interquartile range) & $61(20-$
\end{tabular}

Patient age at Radiofrequency ablation treatment (RFA) (years)

Median (interquartile range)

$67(29-$

86)

Treatment before RFA (per patient)

28 (53)

Interferon

18 (34)

Sunitinib

$9(17)$

Sorafenib

$1(2)$

Primary histology (per patient)

Clear cell carcinoma

$48(90)$

Papillary carcinoma

$1(2)$

Mucinous tubular renal cell carcinoma RCC missing

information

Sarcomatoid component (10\%)

T staging (per patient)

$\mathrm{T} 1-\mathrm{T} 2$

$\mathrm{T} 3-\mathrm{T} 4$

Unknown

$1(2) 1(2)$

$2(4)$

19 (36)

$25(47)$

$9(17)$

Tumor size in $\mathrm{mm}$ (per lesion)

$$
\begin{aligned}
& <10 \\
& 10<\underline{x}<20 \\
& 20<\underline{x}<30 \\
& \geq 30
\end{aligned}
$$$$
\text { missing }
$$

Time to metastasis (months)

Median (interquartile range)

Number of metastases (per patient)

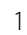

2

3

4-6

Number of RFA treatments (per patient)

1

2

Fuhrman grade (per patient)

1

2

3

4

Unknown
Table 1 Population $(n=53)$ and Tumor $(n=100)$ characteristics (Continued)

\begin{tabular}{ll}
\hline Characteristic & $\mathrm{N}(\%)$ \\
\hline Time to recurrence (all types, per patient) & \\
$\leq 12$ months & $20(38)$ \\
$>12$ months & $33(62)$ \\
Concomitant metastases (per patient) & \\
Concomitant & $10(19)$ \\
Delayed & $43(81)$ \\
\hline
\end{tabular}

\section{Discussion}

In this large study investigating survival after local treatment by RFA for mRCC, good estimated probability of OS of $94,74.5$ and $62 \%$ at 1,3 and 5 years respectively were observed. These rates can be compared with survival rates reported in surgical series after lung metastasectomy for mRCC, with 5-year OS estimated between 30 and $60 \%$ [12-14]. An association between lung metastasectomy and survival was reported [13] but only for patients in the intermediate risk group of the MSKCC prognostic classification [15]. Outcomes after local treatment by RFA of lung metastases have been reported previously on two small series ( 9 and 15 patients treated with curative intent) $[8,9]$. Local efficacy of $91 \%$ and 5-year DFS of 23\% were observed [8]. Our local efficacy, with probability of survival estimated at $91 \%$ is comparable, as is the observed DFS rate of $18 \%$ at 3 years. Until now, the largest published series of lung metastases treated with RFA concerned 566 patients [16] with several primary tumors, including kidney cancer (12\%). The median OS was 62 months and the 4-year local efficacy was $89 \%$.

Local efficacy was evaluated on CT images. Immediately after RFA, the ablation zone appears larger than the original tumor, because it consists of both tumor and perilesional ground-glass opacity corresponding to ongoing necrosis beyond the tumor margins. The enlargement is also explained by consolidation, inflammation, and hemorrhage, after which the ablation area will not increase in size. Then a consolidation will occur during the first 3 months the ablation zone continuing to be larger, compared with the original tumor, but should be smaller relative to the early phase as a result of regressing parenchymal edema, inflammation, and hemorrhage.

Roughly at 3 months, the size of the ablation zone should be larger than the baseline tumor, and by 6 months, the size of the ablation zone should be the same or smaller than the tumor before ablation and in general stable from this period or decreases. After 3 months any increase or appearance of any irregular, nodular, or eccentric focus at the margin of the ablation zone by imaging should be considered suspicious for tumor recurrence. In [16] follow-up evaluation with CT 


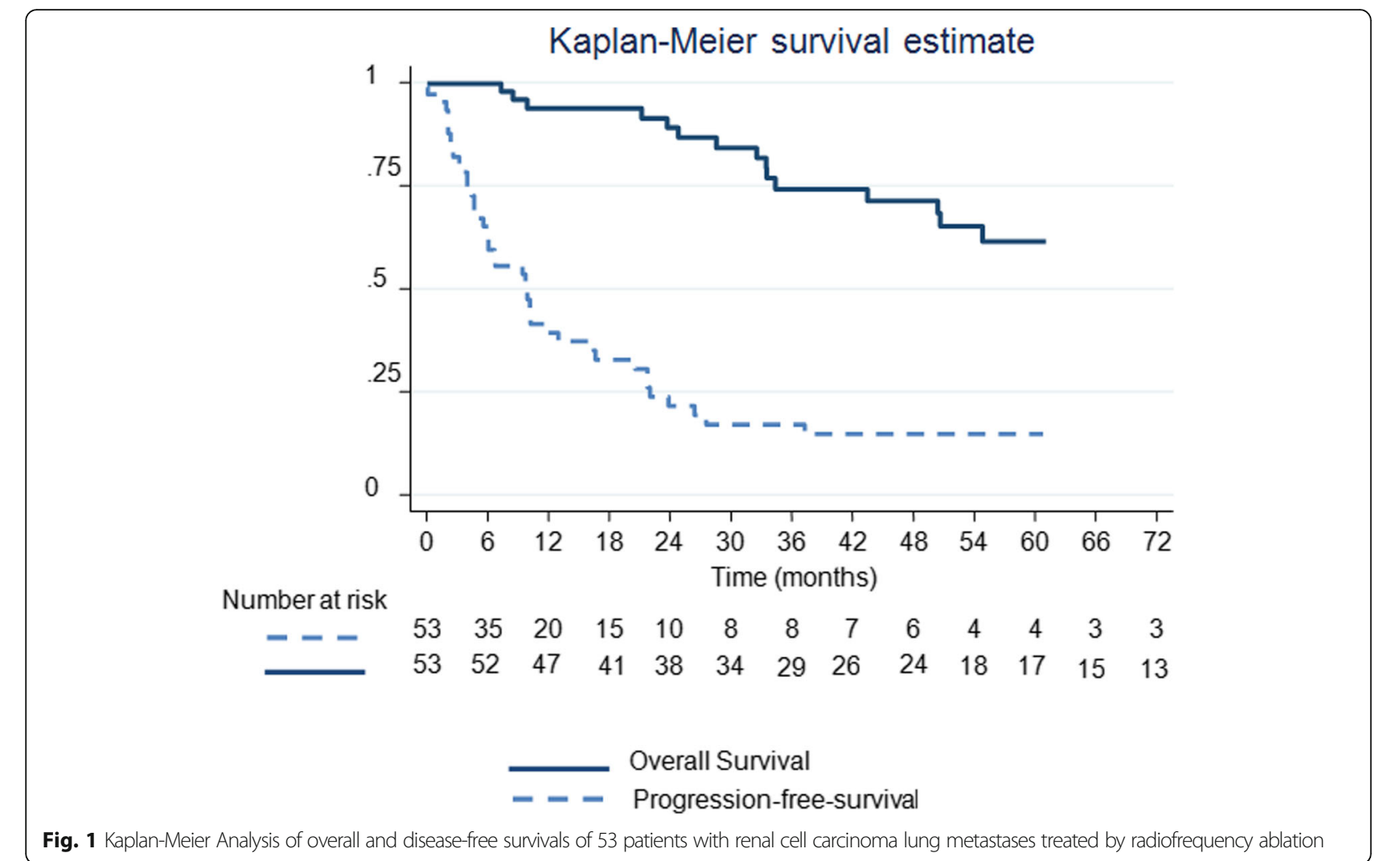

depicted $50 \%$ of cases of local progression during the first year of follow-up. Indeed, among 86 local progressions in 1037 metastases treated with RFA, 54, 21, 5 and 2 were diagnosed during the first, second third and fourth or fifth year of follow-up; reported rates of local tumor progression were $5.9,8.5,10.2$, and $11 \%$ at $1,2,3$, and 4 years, respectively.

Regarding prognostic factors, advanced tumor stage (T3 or 4 of the TNM classification) of the primary RCC was associated with poorer OS, PPFS and STFS. While T-staging is an important prognostic factor for localized RCC [17], this result may reflect the low metastatic load of such a selected population ( $85 \% \leq 3$ metastases). Conversely, Fuhrman grade was not significant, most likely because of patient recruitment for RFA. Only one patient had a Fuhrman grade 1 tumor and five patients had Fuhrman grade 4. Tumor grade is known to be an independent risk factor for recurrence and for tumor migration. There is a low metastatic potential for RCC Fuhrman grade 1 and a higher risk of polymetastatic evolution for Fuhrman grade 4 [18].

Although tumor size of pulmonary metastases $>20$ $\mathrm{mm}$ was associated with poorer $\mathrm{OS}$ in the univariate analyses, this was only of borderline statistical significance in the multivariable model $(P=0.08)$, probably due to limited statistical power. The presence of two or more metastases was associated with poorer PPFS.
Similar findings have been noted in surgical series [3, 12]. This relationship may be explained by an association between the risk of occult disease and the number of metastases [19]. Oligometastasis status is an empiric model [20] describing a biological state before polymetastatic status and so with a limited propensity for metastases. Women were also associated with poorer PPFS.

Time to recurrence was not a significant prognostic factor in our study. While time to recurrence was a criterion of oligometatastic state and prognostic for survival, some publications have reported that synchronous metastases do not have poorer prognosis than metachronous metastases $[3,14]$.

In contrast to previous studies [21] in our series, the presence of extra-thoracic metastases was not associated with survival outcomes. However, even if local treatment is indicated especially in the case of oligometastatic patients, other patients may also receive local treatment for lung metastases. Even for RCC with slow evolution, aggressive metastases with poor prognosis may appear due to clonal diversity [22]. Local treatment of these aggressive metastases may contribute to cancer disease control.

Local treatment has to be considered with systemic treatment strategy. Systemic treatment sparing may offer some advantages, when delayed at initiation [23] or discontinued, both in selected patients. Management of mRCC aims to improve the patients' quality of life 
Table 2 Complications and image findings ${ }^{a}$

\begin{tabular}{ll}
\hline & Number of procedures \\
\hline Pleural effusion & 32 \\
Pneumothorax & 39 (26 drained) \\
Infectious pneumopathies & 4 \\
Pain & 19 \\
\hline
\end{tabular}

anly 3 were Grade 3-4 (pneumothorax requiring prolonged hospitalization; pain limiting daily activities; and a diaphragmatic hernia)

(QoL) while trying to prolong their survival. Targeted agents or immune therapies have improved survival but raise issues relating to the long-term delivery, with $\mathrm{cu}$ mulative toxicity [24] and/or cost. Moreover, treatment discontinuation may reverse resistance to targeted therapies. The occurrence of early progression "rebound effects" has been cited as an argument against treatment discontinuation, but these have only been reported for patients with intermediate or poor prognostic MSKCC group [25]. While from 1.6 to $9 \%$ of complete response (CR) is achieved using the most recent first line systemic treatment [5, 26], partial response (PR) is more frequent and suggests a place of local treatment to make patients disease-free. One advantage of RFA is the ability to repeat local treatment to obtain total tumor clearance from the lung through repeated procedures, and may then answer to the needs of systemic treatment sparing in selected patients: 1-delay treatment initiation, 2-help to achieve $\mathrm{CR}$ in order to discontinue systemic treatment in case of PR, 3-prolong off period of systemic treatment. The concept of oligo-recurrence relates to patients with 1-5 metastatic or recurrent lesions that can be cured by local therapy with controlled primary lesions [27]. This suggests that a rigorous, repeated and

Table 3 Factors associated with overall, disease-free, pulmonary progression-free, and systemic treatment free survivals after radiofrequency ablation of lung metastases from renal cell carcinoma $(n=53)$

\begin{tabular}{|c|c|c|c|c|c|c|c|c|}
\hline & \multicolumn{2}{|c|}{ Overall Survival } & \multicolumn{2}{|c|}{ Pulmonary Progression-free survival } & \multicolumn{2}{|c|}{ Systemic Treatment-free survival } & \multicolumn{2}{|c|}{ Disease-free survival } \\
\hline & Univariate & Multivariable & Univariate & Multivariable & Univariate & Multivariable & Univariate & Multivariable \\
\hline & $P$ (Log-Rank) & $H R(95 \% \mathrm{Cl})$ & $P$ & HR $(95 \% \mathrm{Cl})$ & P & $P$ & & $P$ \\
\hline \multicolumn{9}{|l|}{ Sex } \\
\hline Male & 0.94 & - & & 0.31 & 0.57 & - & 0.53 & - \\
\hline Female & & & & $2.7(1.04-7.2)(P=0.042)$ & & & & \\
\hline \multicolumn{9}{|l|}{ Age at RFA } \\
\hline$\leq 60$ years & 0.67 & - & 0.44 & - & 0.66 & - & 0.94 & - \\
\hline \multicolumn{9}{|l|}{$<60$ years } \\
\hline \multicolumn{9}{|c|}{ Concomitant metastases } \\
\hline Yes & 0.87 & - & 0.85 & - & 0.71 & - & 0.81 & - \\
\hline \multicolumn{9}{|l|}{ No } \\
\hline \multicolumn{9}{|c|}{ Extra-thoracic metastases } \\
\hline Yes & 0.86 & - & 0.73 & - & 0.79 & - & 0.72 & - \\
\hline \multicolumn{9}{|l|}{ No } \\
\hline \multicolumn{9}{|l|}{ T staging } \\
\hline $\mathrm{T} 1-\mathrm{T} 2$ & 0.031 & 1 (Ref.) & 0.024 & 1 (Ref.) & 0.047 & 1 (Ref.) & 0.07 & - \\
\hline T3-Т4 & & $\begin{array}{l}3.55(1.05-12.1) \\
(P=0.04)\end{array}$ & & $3.5(1.3-9.3)(P=0.011)$ & & $2.3(1-5.5)(P=0.053)$ & & \\
\hline \multicolumn{9}{|l|}{ Fuhrman } \\
\hline $1-2$ & 0.31 & - & 0.49 & - & 0.26 & - & 0.71 & - \\
\hline \multicolumn{9}{|l|}{$3-4$} \\
\hline \multicolumn{9}{|l|}{ Lung mets size } \\
\hline$\leq 20 \mathrm{~mm}$ & & 1 (Ref.) & 0.73 & - & 0.17 & - & 0.39 & - \\
\hline$>20 \mathrm{~mm}$ & 0.019 & $\begin{array}{l}2.56(0.88-7.5) \\
(P=n s)\end{array}$ & & & & & & \\
\hline \multicolumn{9}{|c|}{ Number of lung metastases } \\
\hline 1 & 0.69 & - & 0.007 & 1 (Ref.) & 0.60 & - & 0.14 & - \\
\hline 2 & & & & $3.09(1.1-8.7)(P=0.032)$ & & & & \\
\hline 3 & & & & $3.4(1.3-9)(P=0.013)$ & & & & \\
\hline
\end{tabular}


comparative imaging surveillance is warranted. In our series, $13(25 \%)$ patients could benefit from a new procedure when it was possible for lung recurrence. We observed a low rate of complications (3\% grade $3 / 4)$. Due to the risk of pneumothorax, lung RF ablation was not an outpatient procedure. RFA does not impair lung function [7], was easily reproducible and well accepted, avoiding some of the morbidity associated with a repeat thoracotomy. A retrospective study [28] investigated discontinuation of targeted therapy in $\mathrm{mRCC}$ who achieved $\mathrm{CR}$ with either targeted therapy alone or a combined approach of additional resection of residual metastases. The median time without targeted therapies was 7 months. Another retrospective study reported a median time from CR to relapse of 7.9 and 8.2 months with or without local treatment [29]. In our series, 42 patients presented with progression in a median time interval of 8.5 months. Of them 24 resumed ST in a median time interval of 14.1 months. Median STFS was 28.3 months. To repeat local treatment may offer durable CR, and is an alternative to the resuming of ST and may be proposed as long as possible in patients with low-volume metastatic disease. RFA is not limited by the number of lung metastases, since a patient with 23 lung metastases has been successfully treated with RFA over a 10-year period, without impending lung function [30]. Moreover, like with radiotherapy, there is increasing interest in the abscopal and immunologic effects following thermal ablation [31]. With the use of newer targeted agents and immunomodulatory agents, optimization of patient selection through sequencing and combining the various treatment options is challenging.

Nine of our patients underwent a previous thoracotomy before RFA for lung progression. As for liver metastases, it is possible to combine RFA and surgical procedures to treat lung metastases completely. It is however difficult to obtain local control for central metastases because of the heat-sink effect and surgery is a better alternative, allowing lymph node resection. RCC is at risk of lymph node involvement, with an estimated prevalence of around 30\% [32], and a mediastinal location in half of the cases. Mediastinal and hilar lymph node metastases significantly correlate with decreased survival [33]. Systematic lymphadenectomy provides valuable information on staging and prognosis in patients with pulmonary metastasis. In these locations, stereotactic radiotherapy is also an option for non surgical patients [34].

This study has some limits, mainly due to its retrospective design and evolutions in practice over the 11 years of the study e.g. treatment as well as prognostic classifications. In particular obtaining the Heng classification [35] was not possible retrospectively. Prognosis has evolved over this time, due to the development of new systemic treatments (with $53 \%$ of our patients receiving previously-used drugs of immunotherapy or target therapy before RFA). It is difficult to separate the effects of local RFA and systemic treatment. That biopsies were not systematically carried out for histological confirmation is another limitation.

\section{Conclusions}

In conclusion, RFA is an effective and safe treatment of lung metastases from RCC, and it is a therapeutic option for local treatment of patients with or without comorbidities. Combined or sequential systemic therapy and local treatment enables good survival with the possibility of complete remission, with the possibility of delaying the need for systemic treatment for a long time. RFA is a good alternative to surgery offering low-morbidity and the possibility to repeat local treatment to obtain total tumor clearance.

\section{Abbreviations}

DFS: Disease-free survival; OS: Overall survival; PPFS: Pulmonary progressionfree survival; RCC: Renal cell carcinoma; RFA: Radiofrequency ablation;

STFS: Systemic treatment-free survival

\section{Acknowledgements}

Part of this manuscript has been previously presented as an abstract at 2017 CIRSE meeting: Cardiovasc Intervent Radiol (2017) 40(Suppl 2): 25. https://doi. org/10.1007/s00270-017-1725-y

The authors thank Laurence Virmoux for statistics and data collection, Adeline Giraudon for help in drafting the article and Pippa McKelvie-Sebileau for medical editing services.

\section{Authors' contributions}

Study concepts: JP, AG, AR; Study Design: JP, AG, VB; Data acquisition: JP, AG, LS, MGG, XB, GR, VC; Quality control of data and algorithms: VB, AG, JP; Data analysis and interpretation: AG, JP, VB; Manuscript preparation and editing: $A G, J P, V B, A R$. All authors read and approved the final manuscript.

\section{Funding}

Not applicable.

\section{Availability of data and materials \\ The datasets used and/or analysed during the current study are available from the corresponding author on reasonable request.}

\section{Ethics approval and consent to participate}

Informed consent was not required for this retrospective study. Ethics approval was given by the Institutional Ethics Review Board.

\section{Consent for publication}

Not applicable.

\section{Competing interests}

The authors declare that they have no competing interests.

\section{Author details}

'Department of Radiology, Institut Bergonié, 229 cours de l'Argonne, 33000 Bordeaux, France. ${ }^{2}$ Department of Radiology CH Pau, 4 Boulevard Hauterive, 64000 Pau, France. ${ }^{3}$ Department of Medical Oncology, Hospital Saint-Andre, Univ. Bordeaux, 33000 Bordeaux, France. ${ }^{4}$ Department of Medical Oncology, Institut Bergonié, 33000 Bordeaux, France. ${ }^{5}$ Department of Statistics, Institut Bergonié, 33000 Bordeaux, France. 
Received: 28 April 2019 Accepted: 7 November 2019

\section{0.0}

\section{References}

1. Ljungberg B, Campbell SC, Choi HY, Jacqmin D, Lee JE, Weikert S, et al. The epidemiology of renal cell carcinoma. Eur Urol. 2011;60(4):615-21. https:// doi.org/10.1016/j.eururo.2011.06.049 Epub Jul 5.

2. Siegel R, Ma J, Zou Z, Jemal A. Cancer statistics, 2014. CA Cancer J Clin. 2014;64(1):9-29. https://doi.org/10.3322/caac.21208 Epub 2014 Jan 7.

3. Hofmann HS, Neef H, Krohe K, Andreev P, Silber RE. Prognostic factors and survival after pulmonary resection of metastatic renal cell carcinoma. Eur Urol. 2005;48(1):77-81; discussion -2. doi: https://doi.org/10.1016/j.eururo. 2005.03.004. Epub Mar 20.

4. Choueiri TK, Motzer RJ. Systemic therapy for metastatic renal-cell carcinoma. N Engl J Med. 2017:376(4):354-66. https://doi.org/10.1056/NEJMra1601333.

5. Motzer RJ, Tannir NM, McDermott DF, Aren Frontera O, Melichar B, Choueiri TK, et al. Nivolumab plus ipilimumab versus sunitinib in advanced renal-cell carcinoma. N Engl J Med. 2018;378(14):1277-90. https://doi.org/10.1056/ NEJMoa1712126 Epub 2018 Mar 21.

6. Powles T, Albiges L, Staehler M, Bensalah K, Dabestani S, Giles RH, et al. Updated European Association of Urology guidelines recommendations for the treatment of first-line metastatic clear cell renal cancer. Eur Urol. 2017; 7(17):31001

7. de Baere T, Palussiere J, Auperin A, Hakime A, Abdel-Rehim M, Kind M, et al, Midterm local efficacy and survival after radiofrequency ablation of lung tumors with minimum follow-up of 1 year: prospective evaluation. Radiology. 2006;240(2):587-96. https://doi.org/10.1148/radiol.2402050807.

8. Shu Yan Huo A, Lawson Morris D, King J, Glenn D. Use of percutaneous radiofrequency ablation in pulmonary metastases from renal cell carcinoma. Ann Surg Oncol. 2009;16(11):3169-75. https://doi.org/10.1245/s10434-0090664-5 Epub 2009 Aug 13.

9. Soga N, Yamakado K, Gohara H, Takaki H, Hiraki T, Yamada T, et al. Percutaneous radiofrequency ablation for unresectable pulmonary metastases from renal cell carcinoma. BJU Int. 2009;104(6):790-4. https://doi. org/10.1111/j.464-410X.2009.08459.x Epub 2009 Mar 18.

10. Altoos B, Amini A, Yacoub M, Bourlon MT, Kessler EE, Flaig TW, et al. Local control rates of metastatic renal cell carcinoma (RCC) to thoracic, abdominal, and soft tissue lesions using stereotactic body radiotherapy (SBRT). Radiat Oncol. 2015;10:218. https://doi.org/10.1186/s13014-015-0528-z.

11. Alberti N, Ferretti G, Buy X, Desjardin M, Al Ammari S, Cazzato RL, et al. Diaphragmatic hernia after lung percutaneous radiofrequency ablation: incidence and risk factors. Cardiovasc Intervent Radiol. 2014;37(6):1516-22. https://doi.org/10.1007/s00270-014-0854-9 Epub 2014 Feb 12.

12. Assouad J, Petkova B, Berna P, Dujon A, Foucault C, Riquet M. Renal cell carcinoma lung metastases surgery: pathologic findings and prognostic factors. Ann Thorac Surg. 2007;84(4):1114-20. https://doi.org/10.1016/j. athoracsur.2007.04.118.

13. Eggener SE, Yossepowitch O, Kundu S, Motzer RJ, Russo P. Risk score and metastasectomy independently impact prognosis of patients with recurrent renal cell carcinoma. J Urol. 2008;180(3):873-8discussion 8. https://doi.org/ 10.1016/j.juro.2008.05.006. Epub Jul 17.

14. Kanzaki R, Higashiyama M, Fujiwara A, Tokunaga T, Maeda J, Okami J, et al. Long-term results of surgical resection for pulmonary metastasis from renal cell carcinoma: a 25-year single-institution experience. Eur J Cardiothorac Surg. 2011;39(2):167-72. https://doi.org/10.1016/j.ejcts.2010. 05.021. Epub Jun 29.

15. Motzer RJ, Mazumdar M, Bacik J, Berg W, Amsterdam A, Ferrara J. Survival and prognostic stratification of 670 patients with advanced renal cell carcinoma. J Clin Oncol. 1999;17(8):2530-40. https://doi.org/10.1200/JCO. 999.17.8.2530.

16. de Baere T, Auperin A, Deschamps F, Chevallier P, Gaubert Y, Boige V, et al. Radiofrequency ablation is a valid treatment option for lung metastases: experience in 566 patients with 1037 metastases. Ann Oncol. 2015;26(5): 987-91. https://doi.org/10.1093/annonc/mdv037 Epub 2015 Feb 16.

17. Volpe A, Patard JJ. Prognostic factors in renal cell carcinoma. World J Urol. 2010;28(3):319-27. https://doi.org/10.1007/s00345-010-0540-8 Epub 2010 Apr 3.

18. Pantuck AJ, Zisman A, Belldegrun AS. The changing natural history of renal cell carcinoma. J Urol. 2001;166(5):1611-23.

19. Kendal WS. Oligometastasis as a predictor for occult disease. Math Biosci. 2014;251:1-10. https://doi.org/10.1016/j.mbs.2014.02.006 Epub Feb 19.
20. Weichselbaum RR, Hellman S. Oligometastases revisited. Nat Rev Clin Oncol. 2011;8(6):378-82. https://doi.org/10.1038/nrclinonc.2011.44. Epub Mar 22.

21. Alt AL, Boorjian SA, Lohse CM, Costello BA, Leibovich BC, Blute ML. Survival after complete surgical resection of multiple metastases from renal cell carcinoma. Cancer. 2011;117(13):2873-82. https://doi.org/10.1002/cncr.25836 Epub 2011 Jan 10.

22. Klein CA. Selection and adaptation during metastatic cancer progression. Nature. 2013;501(7467):365-72. https://doi.org/10.1038/nature12628.

23. Park I, Lee JL, Ahn JH, Lee $\mathrm{DH}$, Lee KH, Jeong IG, et al. Active surveillance for metastatic or recurrent renal cell carcinoma. J Cancer Res Clin Oncol. 2014; 140(8):1421-8. https://doi.org/10.1007/s00432-014-1680-9 Epub 2014 Apr 22.

24. Escudier B, Szczylik C, Porta C, Gore M. Treatment selection in metastatic renal cell carcinoma: expert consensus. Nat Rev Clin Oncol. 2012;9(6):32737. https://doi.org/10.1038/nrclinonc.2012.59.

25. Powles T, Kayani I, Sharpe K, Lim L, Peters J, Stewart GD, et al. A prospective evaluation of VEGF-targeted treatment cessation in metastatic clear cell renal cancer. Ann Oncol. 2013;24(8):2098-103. https://doi.org/10.1093/ annonc/mdt130 Epub 2013 Apr 11.

26. lacovelli R, Alesini D, Palazzo A, Trenta P, Santoni M, De Marchis L, et al. Targeted therapies and complete responses in first line treatment of metastatic renal cell carcinoma. A meta-analysis of published trials. Cancer Treat Rev. 2014;40(2):271-5. https://doi.org/10.1016/j.ctrv.2013.09.003. Epub Sep 11.

27. Niibe Y, Chang JY. Novel insights of oligometastases and oligo-recurrence and review of the literature. Pulm Med. 2012;2012:261096. https://doi.org/ 10.1155/2012/261096 Epub 2012 Aug 22.

28. Johannsen M, Staehler M, OhImann CH, Florcken A, Schmittel A, Otto T, et al. Outcome of treatment discontinuation in patients with metastatic renal cell carcinoma and no evidence of disease following targeted therapy with or without metastasectomy. Ann Oncol. 2011;22(3):657-63. https://doi. org/10.1093/annonc/mdq437 Epub 2010 Sep 24.

29. Albiges L, Oudard S, Negrier S, Caty A, Gravis G, Joly F, et al. Complete remission with tyrosine kinase inhibitors in renal cell carcinoma. J Clin Oncol. 2012;30(5):482-7. https://doi.org/10.1200/JCO.2011.37.516. Epub 2 Jan 9.

30. Crombe A, Buy X, Godbert Y, Alberti N, Kind M, Bonichon F, et al. 23 lung metastases treated by radiofrequency ablation over 10 years in a single patient: successful oncological outcome of a metastatic cancer without altered respiratory function. Cardiovasc Intervent Radiol. 2016;39(12):177984. https://doi.org/10.1007/s00270-016-1445-8 Epub 2016 Aug 8.

31. de Baere T, Tselikas L, Deschamps F, Soria JC, Marabelle A. Immunooncology in cancer care is a fantastic opportunity for interventional oncology: IO4IO (interventional oncology for Immuno-oncology) initiative. Cardiovasc Intervent Radiol. 2018;41(6):825-7. https://doi.org/10.1007/ s00270-018-1935-y.

32. Renaud S, Falcoz PE, Olland A, Massard G. Should mediastinal lymphadenectomy be performed during lung metastasectomy of renal cell carcinoma? Interact Cardiovasc Thorac Surg. 2013;16(4):525-8. https://doi. org/10.1093/icvts/ivs534 Epub 2013 Jan 3.

33. Winter H, Meimarakis G, Angele MK, Hummel M, Staehler M, Hoffmann RT, et al. Tumor infiltrated hilar and mediastinal lymph nodes are an independent prognostic factor for decreased survival after pulmonary metastasectomy in patients with renal cell carcinoma. J Urol. 2010;184(5): 1888-94. https://doi.org/10.1016/j.juro.2010.06.096 Epub Sep 17.

34. Wersall PJ, Blomgren H, Lax I, Kalkner KM, Linder C, Lundell G, et al. Extracranial stereotactic radiotherapy for primary and metastatic renal cell carcinoma. Radiother Oncol. 2005;77(1):88-95. https://doi.org/10.1016/j. radonc.2005.03.022 Epub Jun 20.

35. Heng DY, Xie W, Regan MM, Warren MA, Golshayan AR, Sahi C, et al. Prognostic factors for overall survival in patients with metastatic renal cell carcinoma treated with vascular endothelial growth factor-targeted agents: results from a large, multicenter study. J Clin Oncol. 2009;27(34):5794-9. https://doi.org/10.1200/JCO.2008.21.4809 Epub 2009 Oct 13.

\section{Publisher's Note}

Springer Nature remains neutral with regard to jurisdictional claims in published maps and institutional affiliations. 